\title{
The GSM Procedures in an Integrated Cellular/Satellite System
}

\author{
Enrico Del Re, Senior Member IEEE, and Piero Iannucci
}

\begin{abstract}
In an integrated cellular/satellite system for mobile communications a satellite subsystem cooperates with a terrestrial cellular network for providing user services. The paper addresses the problems related of the reuse of the procedures of the GSM cellular standard in an integrated system, allowing at most changes only at the mobile terminal and/or in the satellite network. The results of the study show that most of the GSM procedures can be reused in an integrated system (in a few cases with limited protocol variations) but some others would require modifications to the cellular network. The results of this study shall also be useful for the suitable definition of the protocols of an integrated satellite/terrestrial system for the next generation of mobile and personal communication services.
\end{abstract}

\section{INTRODUCTION}

$\mathbf{T}$ HE INTEGRATED system-Several studies have pointed out the advantages deriving from the cooperation between the cellular network and a satellite system to supply mobile communication services (for instance, [1]-[3]). In short, the satellite and the cellular network have complementary characteristics: the main advantages of the use of the satellite are the enlargement and a completion of the service area, the immediate deployment of the service and the availability of additional capacity; on the other hand, the satellite resources cannot support the same traffic volume as a cellular network.

In an integrated system a suitable terminal (dual mode terminal) can establish a call both on the radio frequency (RF) cellular link and on the satellite link and the network features are designed to take the maximum advantage from the presence of the two subsystems, satellite and cellular. Even if a more refined classification is possible, essentially three approaches can be individuated:

1) the satellite subsystem adopts all the techniques and procedures of the terrestrial one. In particular the same protocols defined for the terrestrial RF link are used on the satellite RF link. Only the RF parts of the mobile terminal equipment must be duplicated, as a specific frequency band should be assigned to the satellite link;

2) the fixed parts of the two sub-networks use the same protocols. Instead, on the two RF links, the reuse is not extended to the lower OSI layers of the RF links: different bit rates, modulations, coding schemes and synchronization procedures can be adopted, taking into

Manuscript received January 15, 1994; revised September 15, 1994. This work was supported by the Italian Space Agency (ASI)

The authors are with the Dipartimento di Ingegneria Elettronica, Università degli Studi di Firenze, 50139 Firenze, Italy.

IEEE Log Number 9408073. account the different characteristics of the two RF channels;

3) the satellite link is based on specific procedures and techniques but it is interconnected to the cellular network.

In this paper we focuse on the first and second approach, considering an integration between a satellite system and the cellular pan-european standard GSM (global system for mobile communications); as a reasonable consequence, we will assume in our study that variations to the infrastructures of the cellular network are not admissible. Changes with respect to the GSM standard, if required, should be limited only at the mobile station (MS) and/or at the ground infrastructures of the satellite subsystem.

The aim of the study is twofold as follows:

1) to determine the feasible degree of reuse, in the satellite subsystem, of procedures designed for a cellular network;

2) to outline techniques that assure good cooperation between satellite and cellular subsystems.

1) Role of the Satellite: The definition of procedures demands to precise the role of the satellite in the integrated system. Basically there are two possibilities that may be exemplified by means of the functioning of the terminal before the set-up of a call as follows.

- A first criterion considers satellite channels equivalent to the terrestrial channels: that is, the choice among global resources (cellular and satellite channels) is carried out considering exclusively link quality parameters: the channel which, at any time, guarantees the best service quality is chosen.

- Another strategy assigns lower priority to the satellite resources: the mobile terminal should use the satellite frequency band only if terrestrial channels are not available. In particular this occurrence should arise in three circumstances:

- when the terminal is outside the coverage of the cellular network;

- when the terrestrial infrastructures are temporarily out of use;

- when phone traffic exceeds the capacity of the cellular network in the area where the mobile terminal is located.

This management criterion recognizes specific features of the satellite resources (more limited, more costly, able to serve a wide area) and it has been assumed as a basic hypothesis for 
the proposals included in this paper. Preliminary studies [2] seem to demonstrate that this strategy assures a considerable improvement of the efficiency of the whole integrated system, with respect to the first assumption.

2) Outline of the Paper: The study considers the main procedures of the GSM network and aims at suggesting similar ones for an integrated system. Sections II provides a brief outline of some GSM concepts and general considerations on the architecture of an integrated system. Section III deals with the activities of the mobile stations (MS's) in idle mode, i.e., the MS's not engaged in a call. The definition of the "paging area" in the satellite environment is considered in Section IV. In Section V we study the possibility of using the GSM frame synchronization techniques on the satellite link. Section VI defines a procedure for the set-up on the satellite link when the cellular channels are busy. The subject of Section VII is to investigate the possibility of passing a call from the satellite subsystem to the cellular network or vice versa. Eventual considerations will be resumed in Section VIII.

\section{BACKGROUND AND GENERAL CONSIDERATIONS}

1) The GSM Architecture: The GSM is a cellular system, so the service area is divided in cells, each one with a preassigned set of radio channels. Each cellular site is covered by the antennas of a BS. In the GSM a group of BS's is connected to a mobile switching center (MSC), which manages the links with the public switched telephone networks (PSTN's), the public data networks (PDN's) and other MSC's. Each MSC is supplied with one visitor location register (VLR). A VLR is a database containing the position information of the terminals standing in the area of the MSC. The MS position is individuated by the location area where it is located: A location area is composed by one or more cells. Each GSM national network, i.e. each public land mobile network (PLMN) has its own home location register (HLR), where information about all the customers of that network is stored. In particular, in the HLR, the VLR where each customer is temporarily located is registered.

2) The GSM Radio Interface: The GSM adopts the digital modulation GMSK and a time division multiple access (TDMA) scheme on a number of carriers spaced $200 \mathrm{KHz}$ apart. In every cell the transmissions on the uplink (from MS to BS) and the downlink (from BS to MS) occur on two different bands, each one comprising several carriers (Fig. 1). An uplink carrier is uniquely associated with a downlink carrier.

The GSM distinguishes between physical channels (i.e. the timeslots) and the logical channels (i.e. the information carried by the physical channels). The TDMA frame arrangement of the GSM is quite complicated. The basic element is a frame of eight slots. The frames are organized in a more complex structure called hyperframe. Each call is assigned a defined timeslot, in which a dedicated traffic channel (TCH) and two dedicated control channels (FACCH and $\mathrm{SACCH}$ ) are multiplexed (TCH, FACCH and SACCH are examples of logical channels). The transmission can be performed with a frequency hopping option: in this case the frequency carrier varies frame to frame according to a prescribed algorithm.

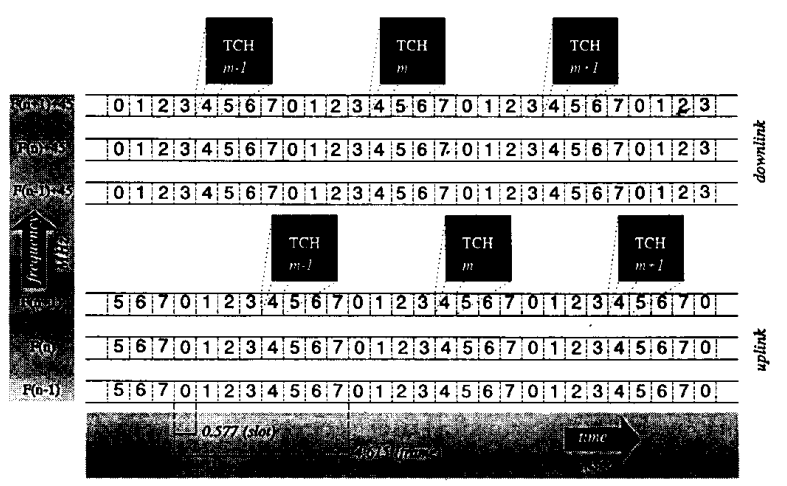

Fig. 1. GSM radio interface. A BS site with three carriers is shown; numbers distinguish the slots of the frames; note that the downlink frames are advanced by three timeslots with respect to the uplink frames; in this example slot 4 of the carrier $F(n+1)$ (and slot 4 of the associated downlink carrier) is assigned a TCH; if frequency hopping were active, the TCH would change frequency frame-to-frame.

Common control channels are foreseen: in particular, on the downlink, every BS broadcasts the BCCH on a fixed timeslot and a constant frequency, by which a set of parameter, related to the cell, is communicated to the MS's in idle mode. The $\mathrm{BCCH}$ is multiplexed with the paging channel, $\mathrm{PCH}$, the frame synchronization channel, $\mathrm{SCH}$, and the channel for the carrier recovery, $\mathrm{FCH}$. The timeslots of the $\mathrm{BCCH}$ on the uplink are reserved for the $\mathrm{RACH}$, the channel by which the mobile terminals asks for a dedicated channel.

At the BS, the uplink and downlink slots are aligned (that is they begin at the same instant), but the downlink frames are advanced by three timeslots with respect to the uplink frames.

3) Reference Architectures and Management Criteria: The definition of procedures for the integrated system depends on the architecture of the satellite subsystem and the management criteria of the whole network.

2. A first distinction among satellite architectures is based on the orbit type:

1) geostationary;

2) low, medium or elliptical.

Architectures of the first type can be implemented with one satellite; low, medium or elliptical orbits need a constellation of satellites to assure the continuity of service and procedures for handing over a call from a satellite to another must be implemented. The discussions about techniques to implement an inter-satellite handover are still at a primary stage. For simplicity, when it will be required to precise the orbit type, only the geostationary case will be considered. Two main drawbacks related to this choice are related to the round trip delay and MS power requirements. Preliminary assessments of the link budget are reported in [1], in the cases of 7 and 12 spots covering Europe. Results show that link budget is satisfied at least for a transportable terminal. The implementation of a hand held terminal seems rather demanding and a lower bit rate might be necessary. Concerning the round trip delay, a preliminary check has been carried out on layer 3 GSM procedures. These 
signals are controlled by timers, supervising that the time between the sending of a certain message and the receiving of another prescribed message does not exceed a specified value. We checked that the specified time-out values are compatible with the round trip delay on the satellite link. Instead, the effects of this delay on lower layers timings require a more accurate analysis and will be considered in Section V.

$\checkmark$ The reference architectures of the satellite subsystem have one or more beams on the feeder link [to/from satellite from/to fixed earth stations (FES's)] and multiple beams on the mobile link (to/from satellite from/to mobile terminals). Two cases may be distinguished:

1) one FES per spot: every spot on the mobile link is covered by only one FES; that is the whole band of the beam is allocated to one FES.

2) several FES per spot: on the mobile link, the frequency band of each beam is shared among several FESs.

In both cases one FES may have a band in several beams of the mobile link.

- Of course, the terrestrial infrastructures of the cellular network coincide with the GSM system. Access points of the satellite network are SATellite gateway MSC's (SAT-MSC's). Each SAT-MSC has its own SATellite $V L R$ (SAT-VLR) and may serve one or more FES's. Essentially, the FES's act as BS's of a cellular network. In principle, even a more simplified structure is possible, with the FES's directly connected to the GSM MSC's. In this case, however satellite subsystem have to adopt a GSM interface between FES's and MSC's and this consequence represents a further constraint.

- A classification of the integrated system based on management criteria can include two classes:

$A$ - the satellite subsystem implements an "autonomous" network, that is logically and functionally equivalent to a PLMN, added and overlaid to the existing terrestrial PLMN's;

$B$ - the satellite spot beams covering the same areas of a terrestrial PLMN are logically and functionally considered as macrocells of that PLMN.

These four classifications will be useful later on. Considerations referring to a specific classification will be preceded by the corresponding symbol $(\boldsymbol{\phi}, \diamond, \boldsymbol{\phi}, \boldsymbol{\bullet})$.

\section{Cell or Beam Selection}

1) Selection and Reselection in the GSM: In the GSM, when the MS is in idle mode, it carries out the selection and reselection procedures. The selection is performed after the switch-on or the re-entrance into the coverage area of the system. The terminal makes out a list of BCCH carriers, evaluates the received signal power and considers the value of the parameter cell_barr_access, that, if set, classifies the cell as "not allowed". The reselection is a routine operation carried out periodically when the MS is on and inside the cellular radio coverage. This operation is performed by the terminal considering a $\mathrm{BCCH}$ allocation list, broadcasted by the selected $\mathrm{BS}$ on the $\mathrm{BCCH}$. In this list, the $\mathrm{BCCH}$ carrier frequencies of the neighboring cells are indicated.

When the MS is required to supply a service, it accesses the network in the cell selected at the time: in the set-up of an MS terminating call, the terminal replies to the paging message sent by the BS of the selected cell; in the set-up of an MS originated call, the MS sends the access bursts on the RACH of the selected cell.

\section{A. Selection and reselection in the integrated system}

I) Spot Beams as Macrocells: In all the reference architectures the spot beams are considered as macrocells and the MS can perform the selection and reselection procedures considering the satellite frequency band in addition to the cellular one.

2) Selection: At the start of a selection procedure the MS operates as in the GSM system, searching for a suitable cell in every allowed terrestrial PLMN. If the search is unsuccessful, a similar procedure is activated on the satellite frequency bands.

$\nabla:$ To reduce charge in terrestrial tails, when the frequency band of the beams are shared among different FES's, it is suitable that the MS checks first the band which is allocated to the FES nearest to the area which it communicates more frequently with. When the mobile terminal switches to the satellite band, therefore, the MS have to checks first 'its own' frequency band: this requires for the mobile terminal to keep in its memory information about "its" BCCH satellite carriers.

2) Reselection: If the MS has initially selected a GSM cell, it performs the reselection procedure in the cellular network. Otherwise, if the MS has initially selected a spot beam, the reselection might be performed among other beams with the same procedure. The $\mathrm{BCCH}$ allocation list, transmitted on the satellite $\mathrm{BCCH}$, refers to the satellite frequencies and must identify the $\mathrm{BCCH}$ carriers in the bands assigned to the beams. This method requires very few modifications to the GSM protocols. The drawback is that, if the MS initially has selected a spot, it will use a satellite channel during a call even if a GSM channel becomes available. So it is necessary for the MS to check periodically the availability of terrestrial channels and the most suitable procedure, when the terminal has initially selected a beam, seems the following:

- the MS performs reselection activity only among beams;

- at a regular intervals, it breaks off this activity and starts a selection procedure. The selection should be carried out on the PLMN's which have cells in the zone where the MS is located. The list of these PLMN's can be filled and updated by the mobile during the same selection procedures. Since the purpose of the periodic selection activity is to detect a suitable cell, it can be limited at the GSM band.

\section{LOCATION OF THE MOBILES}

1) Location Area: In the GSM, the smallest area unit used for the location of the MS is called location area (LA). A LA is composed by one or more BS areas and is identified in a PLMN by a LA Identification (LAI), which is a parameter broadcasted on the BCCH. When the MS selects a new cell, it looks for the LAI. If the new LAI is different from the 
one of the previous selected cell, it starts a location updating procedure.

$\checkmark$ : We can assign a location area identification to every spot beam or to the whole satellite network. In any case, in the areas covered by the GSM and the satellite, the LA's of the two systems are overlaid. The location area identification is composed by a set of bits, subdivided into the following fields:

1) 3-digit mobile country code (MCC), that identifies the PLMN country

2) 2-digit mobile network code (MNC), that identifies a PLMN within a country (multiple GSM operators are foreseen in a country)

3) location area code (LAC) (with a length not exactly defined but not greater than 2 bytes), that identifies a LA within a PLMN.

In type $\mathrm{A}$ architectures, if we assume that there is one international satellite system, it is possible to assign it a specific MCC: this is sufficient to distinguish between the terrestrial LA's and satellite LA's.

If each country has its own satellite network, the spot beams belonging to a country have the national MCC. However, it is possible to distinguish between the LA's of the two systems by the MNC: the national satellite system operator should have a MNC different from the MNC's of the cellular operator.

In type $B$ architectures, the distinction between satellite LA's and terrestrial LA's can be assured by the LAC's.

$\nabla$ : Let us suppose an FES has a band in more than one spot. In this case the LA is a concept that must be associated with the FES and not with the spot area. The spots where a particular FES has a frequency band can be considered, for that FES, as one LA or can be grouped in various LA's. Obviously each spot will be included in several LA's, if different FES's have frequency bands in this beam.

\section{SYNCHRONIZATION}

1) Frame Synchronization in the GSM: The GSM frame synchronization distinguishes three levels.

1) The uplink and downlink frames are cyclically num-

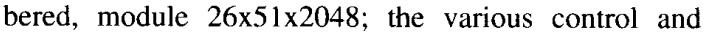
traffic channels are defined and communicated by the BS to the MS's with reference to the frame number. The frame number is sent on the $\mathrm{BCCH}$ carrier every ten frames.

2) Each frame is divided in 8 slots; each MS have to know the timeslot number either when it is in idle mode or it is communicating with the network. In idle mode the MS have to ascertain the instants at which it may send an access burst and have to determine the time for listening to the paging channel. During a call, the mobile has to send and receive the bursts within the assigned timeslots.

3) While a conversation is in progress, the bursts sent on the uplink have to arrive to the FES-BS aligned with the initial instant of the slot assigned to that communication, so that they do not overlap to the adjacent slots. In the GSM system the BS sends to each MS that has a call in progress the timing advance parameter, whose value depends on the estimated delay on the link BS-
MS-BS. The MS anticipates its transmissions by this value, in order that the bursts from different MS's are compensated for the delay at the BS and are correctly positioned in the assigned time slots. This technique, called adaptive frame alignment, needs a more accurate description.

2) Adaptive Frame Alignment: The frame transmitted by the BS is received by the MS with a propagation delay Tbm. In the access phase the MS does not take into account this delay and synchronizes with the received frame, sending the random access burst at the beginning of a RACH slot. This signal arrives at the BS delayed by Tmb. The random access burst is sufficiently short, such as, even if delayed by Tbm+Tmb, it remains within the assigned slot (it does not overlays to the following slot). The GSM has an access burst of $88 \mathrm{~b}$ $(0.325 \mathrm{~ms})$ and, therefore, a guard time of 68.25 bits $(0.252$ $\mathrm{ms})$. Consequently $\mathrm{Tbm}+\mathrm{Tmb} \leq 0.252 \mathrm{~ms}$. Assuming the two delays equal to $\mathrm{Tr}$, we have that $\operatorname{Tr}_{\max }=0.126 \mathrm{~ms}$, which corresponds to a maximum BS-MS distance of about $37.7 \mathrm{~km}$. The maximum cell radius, in the GSM, is $35 \mathrm{~km}$. This delay, rounded to the nearest bit period, is called timing advance (TA) and is transmitted to the MS. The maximum permitted value for the timing advance is $63 \mathrm{~b}$. The MS synchronizes its transmissions accordingly: it waits for the reception of the allocated timeslot number on the uplink and transmits its burst with a delay equal to 468.75 -TA bit periods, i.e., three timeslots duration minus TA. The BS continues monitoring the delay of the received signal and, if it detects a variation greater than one bit period with respect to the current timing advance, the parameter TA is increased or decreased by 1 and signaled to the MS.

\section{A. Synchronization on the satellite link}

1) Delay and Spot Size: Two aspects of the satellite link have to be taken into account:

- the higher propagation delay than in the cellular link. For a geostationary satellite, the only case here considered, the propagation delay is of the order of $250 \mathrm{~ms}$; this value should be compared with the maximum one admitted by the GSM $(0.126 \mathrm{~ms})$;

- the much higher size of the spot beam with respect to the cell size; as a consequence the values of the roundtrip delay vary in a range much wider than those involved in a GSM cell $(0.252 \mathrm{~ms}$ maximum, corresponding to the guard time of the access burst).

2) Delay Evaluation: After receiving the access burst from the MS, the FES-BS must compute the delay of the received message. If we wish to adopt the same method of the GSM to evaluate this delay, it is convenient to assume that a carrier is reserved for the access in the spot beam (access carrier). On this carrier, the MS should be allowed to send access bursts only at specified time instants. To define the minimum time interval between these instants, it is convenient to consider an example (Fig. 2). Assuming a spot beam with a radius not greater than $800 \mathrm{~km}$, the difference between the maximum single hop delay (when the MS is at the maximum distance from the satellite) and the minimum single hop delay (when 


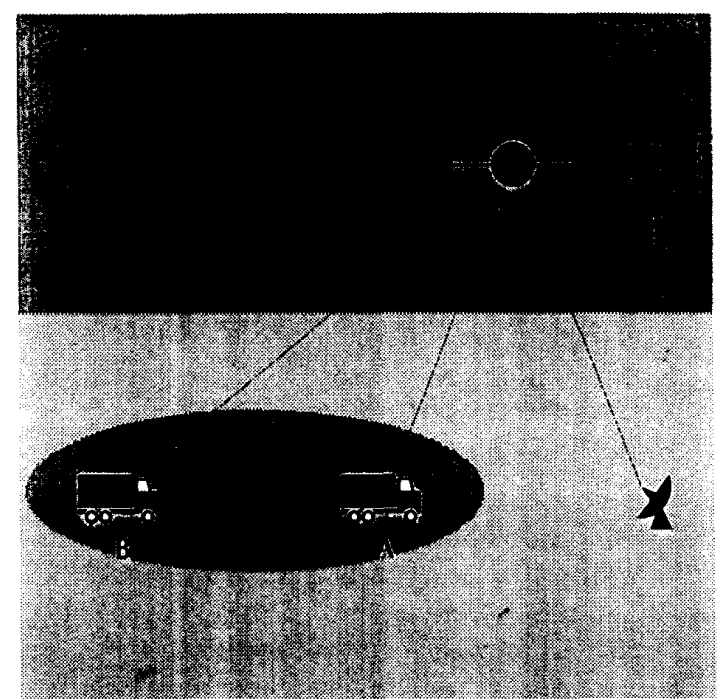

Fig. 2. Delay in the spot footprint.

the MS is at the minimum distance from the satellite) is less than eight timeslots. So, two MSs perceive the same "allowed instant," on the downlink, with a time difference T, less than eight timeslots. If both transmit the access burst when they perceive the "allowed instant," the time difference with which these bursts will be received at the FES is $2 \times \mathrm{T}$, not greater than 16 timeslots. In this case a suitable choice of the interval between two "alloved instants" is 16 timeslots. At the FES, to determine the round trip delay, it is sufficient to detect the arrival instants of the access bursts in an interval of 16 timeslots. With a spot beam larger than the previous one, this interval must be increased accordingly.

The evaluation of the access burst delay can guarantee that the successive bursts, transmitted by an MS, arrive at the FES-BS correctly positioned within the uplink timeslots, in the assigned slot and in the specified frame. To understand how this is possible, a discussion on the staggering between the uplink and downlink frame number is needed.

3) Staggering Between Uplink and Downlink Frames: In the GSM, at the BS, the downlink and uplink frames are aligned (same frame number), with downlink frames advanced by three timeslots with respect to uplink frames. A similar situation occurs at the MS, where the uplink frame is delayed by ( 3 timeslots)-(timing advance) with respect to the received frame. In other words, apart from the shift of three timeslots (or about three timeslots) on uplink and downlink, at the BS and at the MS's, the current frame number is the same. On the satellite link, this coincidence of frame numbers is not possible because of the delay which is of the order of 55 frames (single hop). Two solutions are possible to accommodate this shift of the frame numbers.

1) Uplink and downlink frame numbers coincide at the FES-BS; at the MS, instead, the current frame number on the downlink is less than the current frame number on the uplink [Fig. 3(a)]. Indicatively, the value of the staggering is about 110 frame periods: the actual

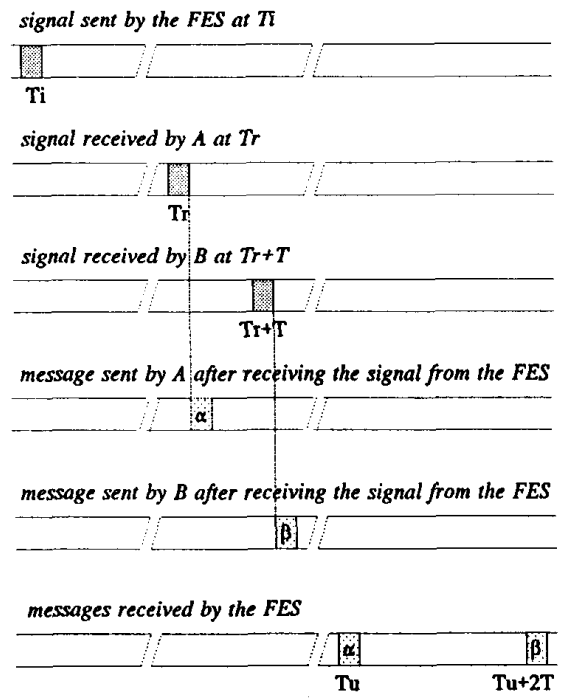

staggering depends on the position of the terminal inside the spot, being greater for the MS farther from the satellite.

2) The downlink and uplink frame numbers coincide for an MS at the maximum distance from the satellite [Fig. 3(b)]. Consequently, at the FES-BS, downlink frame number has to be in advance with respect to the uplink one. The staggering that must be accommodated at the FES-BS, has to be not less than twice the maximum single hop delay in the spot footprint.

In both cases, the staggering at the MS depends on the position of the terminal inside the spot footprint. The FES-BS, after evaluating the delay of the access burst, can communicate the MS the staggering it has to adopt.

4) Start of the Transmission: To assure the correct synchronization of the bursts received from the MS, the FES-BS can informs the terminal about two quantities: the staggering between the uplink and downlink and the assigned timeslot. The communication of the data could be carried out with the same GSM signaling. In reply to an access burst the BS sends the immediate assignment message, where the timing advance ( 8 information bits) and, optionally, the starting time (16 information bits) are indicated. The fields assigned to these parameters could be used by the FES-BS to inform the MS of the timing for the initial synchronization. In general the staggering is not equal to an integer periods of frame; the fractional part can be expressed in number of slots and number of bits. To indicate the number of frames $7 \mathrm{~b}$ are sufficient, as the frame number difference is of the order of 110; however, we could use less bits, assuming to indicate only the variation with respect to the minimum staggering. For the number of slots, $3 \mathrm{~b}$ are sufficient, as its range is 0 to 7. The number of bits requires $8 \mathrm{~b}$, as the GSM slot includes $156.25 \mathrm{~b}$. In this way the fields of the immediate assignment message are sufficient to provide all the information for the initial synchronization. 


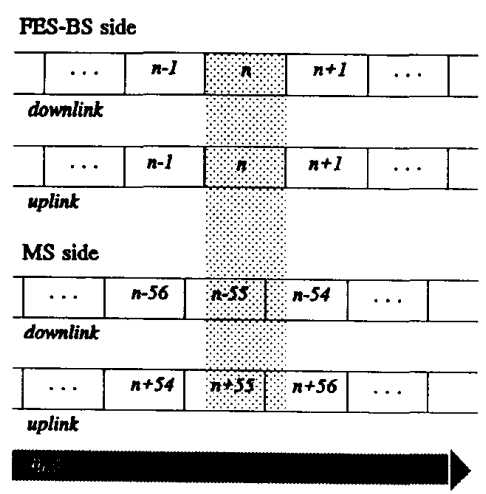

(a)

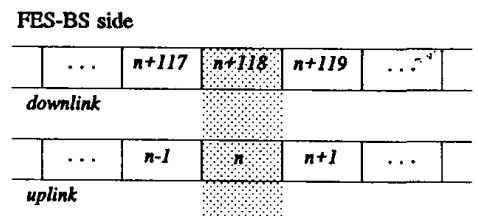

MS side (at the maximum MS-satellite distance)

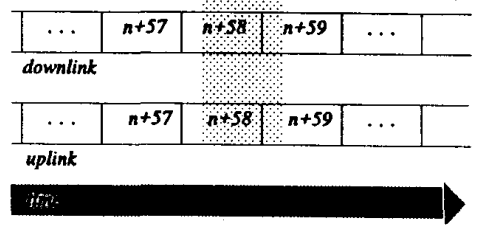

(b)

Fig. 3. Staggering between uplink and downlink frame numbers on the satellite link.

Moreover, we have to take into account that in the GSM system all the codes for the parameter identification are not used; thus some codes could be used for the definition of parameters for the synchronization in the satellite network.

5) Access Carrier: The method described above is based on the existence of access carriers. It is necessary to assess if this assumption fits the GSM constraints on the RF link. Fig. 4 shown a possible combination of the $\mathrm{BCCH}$ channel with other logical channels. We recall that all the possible combinations that include the $\mathrm{BCCH}$ cannot undergo to the frequency hopping. The uplink carrier of this configuration might be used as the access carrier: in effect one of the 8 timeslots of the TDMA frame is already assigned to the $\mathrm{RACH}$. The remaining 7 timeslots of that particular uplink frequency cannot be used for other channels. Consequently, on the downlink, in these 7 timeslots the FES-BS must transmit dummy bursts, specified in the GSM system for similar cases: they are transmitted by the BS in the timeslots of the BCCH carrier not assigned to any communication to hold constant the power level transmitted on this frequency.

Note that the unavailability of some timeslots does not require to modify the frequency hopping algorithm: as the hopping frequencies are indicated to the mobile with the cell allocation list, included in the message immediate assignment, it is sufficient for the carrier access frequency not to be included in that list. However some studies [4] report that

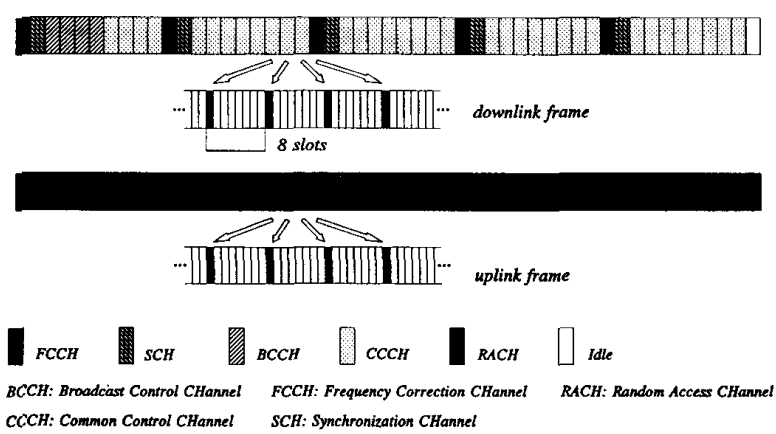

Fig. 4. Combination of the $\mathrm{BCCH}$ with other logical channels.

the system performance does not degrade appreciably if the frequency hopping is not used on this link, due to the different statistical characteristics of the RF satellite channel. The obvious solution is of avoiding the frequency hopping option.

Another possibility is to reserve for the uplink access carrier a frequency, with which no downlink frequency is associated. In this case the RACH timeslots of the configurations of the Fig. 4 are not used on the satellite link, as the access to the FES-BS always occurs on the assigned frequency.

6) Adaptive Frame Alignment on the Satellite Link: Till now, all the questions that have been discussed with respect to the synchronization on the satellite link are related to the initial access. The remaining aspect to be examined is the adaptive correction of the synchronization during a call. Referring to a geostationary orbit and disregarding the satellite position shifts, the problems that arise are the following ones:

- the MS position is determined by the FES with a delay of about $250 \mathrm{~ms}$;

- the MS receives the new timing advance with a delay of about $250 \mathrm{~ms}$.

The synchronization correction is carried out with a further error, with respect to that one which may arise in the GSM, due to the distance covered by the mobile in about $500 \mathrm{~ms}$. If we assume a speed of $250 \mathrm{~km} / \mathrm{h}$ for the terminal, this distance is $34.41 \mathrm{~m}$. This value causes an error about the distance earthsatellite-FES that, even for very critical cases, is less than 30 $\mathrm{m}$ : its effect on the timing advance is negligible.

7) Duplexer: A staggering of three timeslots between uplink and downlik frames is used in the GSM to have a time separation of transmission and reception and, consequently, to avoid the use of a duplexer. At the MS, even if the staggering is smaller due to the timing advance, it still separates the transmission and reception intervals sufficiently. In the satellite network, on the contrary, at the MS this separation cannot be guaranteed as the MS could be in a position where it must transmit while it is waiting for a slot on the downlink. Therefore the mobile terminal for the integrated system must be equipped with a duplexer.

\section{SET-UP}

1) Traffic Overload: In addition to providing services to areas not covered by the terrestrial infrastructures, one of the most attractive features of the dual mode terminal should be 


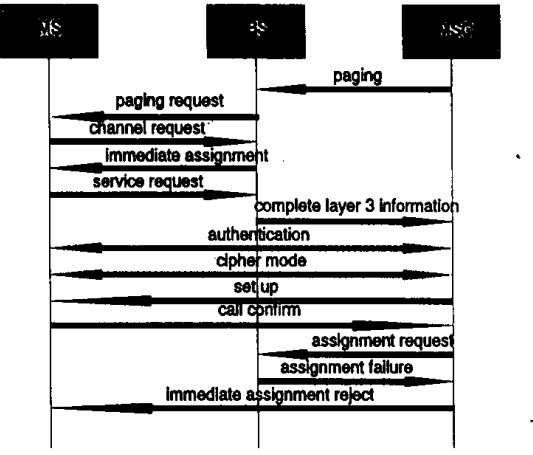

(a)

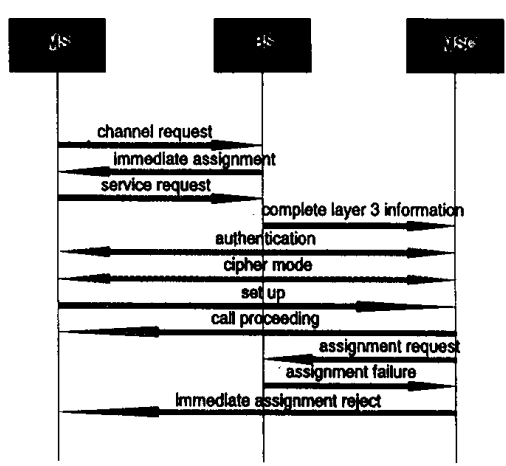

(b)

Fig. 5. Call reject: (a) mobile terminating call; (b) mobile originated call.

the possibility of establishing a call through the satellite link when a traffic overload arises in the cellular link. A congestion situation is reported by the BS to MSC with the message assignment failure. Receiving this message, the MSC breaks off the normal set-up. The succession of messages till the assignment failure is showed in Fig. 5(a) (mobile terminating call) and Fig. 5(b) (mobile originated call). From now on we shall define a set-up procedure for a dual mode terminal.

2) Set-Up in a GSM Cell: Let us suppose that the MS has selected a GSM cell and that the set-up is not completed owing to a congestion in this cell. It is suitable to distinguish two cases:

1) mobile terminating call: the mobile is the called MS, that is the traffic overload occurs in the "destination" cell. A procedure for trying an alternative routing through the satellite network is not advisable as, to implement it, modifications should be taken into account in the terrestrial network. As a matter of fact:

- the MSC that receives the assignment failure is a GSM MSC and in this system a second set-up attempt is not provided;

- the MS wait for the paging message only on the proper channel of the selected cell. A procedure for an alternative routing requires that the MS is registered in two VLR's, one of which belonging to the satellite network. So, the terminal should perform two location updating and listen to two
BCCH. This integrated network should work in such a way that the call is first routed through the terrestrial VLR. A procedure for implementing this set-up is much more complex than the one of the GSM and, in any case, different.

2) mobile originated call: the MS has selected a GSM cell and it is the calling terminal, that is the traffic overload arises in the 'originating' cell. A possible procedure is the following. When the first set-up fails, the MS can choose a new terrestrial cell. If the second attempt also fails, another set-up will be tried, but, this time, on the satellite link. In details the proposed procedure is:

- when the MS infers that it is not possible to establish the call in the selected cell, it chooses a new cell, using the list compiled during the reselection activity. If possible, the new cell should be in the same location area, so that it is not necessary a location updating. It is preferable to try selecting a cell before a beam, as a call through the satellite is much more expensive for the subscriber;

- if the second attempt is not successful, the terminal will ask the subscriber, with a message or another signal, whether he wants to try the set-up on the satellite link;

- if the response is affirmative, the MS will choose a beam and perform a location updating.

3) Set-Up in a Spot Beam: The set-up in a spot beam can be performed with the GSM procedure. Analogously to the case examined in the preceding section, a procedure might be defined for a second set-up if the first one fails. This circumstance occurs when all the spot beam channels are occupied. A second set-up when the MS is the called terminal will demand modifications to the GSM network and, therefore, it is not easily feasible. On the contrary, when a traffic overload occurs in the call originating spot, no change is required in the network. In accordance with the repeatedly recalled management criteria, it would be better for the second attempt to be performed into GSM network. However, if the described reselection procedures are adopted, it is likely that when an MS accesses the satellite link it is outside the GSM coverage. So, if a second set-up has to be attempted, it should again be carried out in the satellite network. Note that if the satellite system is implemented on the basis of the GSM protocols, it is not possible for the MS to know the beam occupancy state before the set-up. It may be proposed to utilize the parameter cell_bar_access of the spot beam as beam channel occupancy indicator: it should be set to 1 when a traffic overload arises. In this way, a useless call set-up is avoided.

\section{HANDOVER}

1) Handing Over a Call in the GSM: In the GSM, the handover can be started by the following.

- Any MSC, which decides to hand over the calls from a cell to the adjacent ones to prevent a traffic overload. The MSC sends to the BS the message handover candidate enquiry, in which the candidate cells are 
characterized by the BSIC (see after) and the BCCH carrier. The BS generates a specific handover required for each MS that can be passed to any indicated cell;

- any BS, when it happens that the quality of the communication goes down prescribed thresholds. This presumably occurs when the MS is leaving the serving cell. The BS sends to its MSC the handover required, containing a list of the candidate cells for the operation.

The decision to start a handover and the continuous updating of the list are carried out by the BS, on the basis of the processing of some measurements performed either by the BS and the MS.

2) Identification of the Neighboring Cells: To handover purposes the MS is in charge of monitoring the $\mathrm{BCCH}$ carriers of the neighboring cells. The $\mathrm{BCCH}$ carriers that have to be monitored by the mobile during a communication, are indicated by the $\mathrm{BS}$ on the $\mathrm{SACCH}$, with the $\mathrm{BCCH}$ allocation list. So it is necessary for the MS to decode the Base Station Identification Code, BSIC. The BSIC is transmitted on the $\mathrm{BCCH}$ and is $6 \mathrm{~b}$ long: 3 bits are allocated for distinguishing among different PLMNs, in a certain area, other 3 bits identify a certain number of cells locally. Given a cell, the BSIC permits to distinguish all the neighboring ones.

3) Handover Procedures: The handover procedure between MSC's is shown in Fig. 6. For simplicity, the MSC handing over the call will be called MSC-A, the other one MSC-B. Receiving the handover required from the BS, the MSC-A chooses a cell among those indicated in the message list. When the MSC-A has identified the new BS, it notifies the involved MSC with the message perform handover. The MSC$\mathrm{B}$ sends a handover request to the new BS which replies with a handover request acknowledge, that specifies the assigned radio resources. The MSC-A sends to the MS, through the BS, the message handover command on the FACCH. The handover command includes, among other things, the description of the new channel and, optionally, the starting time; this is the indication of the frame from which the MS may start transmitting on the new channel. The MS switches to the new channel and sends continuously the handover access bursts, with reduced length, on the new FACCH. After receiving the message physical information from the BS, the MS stops sending access bursts and replies with the handover complete message. The subsequent messages complete the handover procedure, including the release of the old radio channel.

\section{A. The Handover in the Integrated System}

1) Internetwork Handovers: In an integrated system it is interesting to study the feasibility to pass a call in progress from the satellite to cellular network or vice versa. The GSM recommendations does not consider possibility of an automatic handover between two PLMN's. As a consequence, it is not possible to implement a handover procedure between satellite and GSM network in type A architectures. Instead, in type B architectures, this problem can be examined.

2) Handover from a GSM Cell to a Spot Beam: When an MS accesses a new BS, it needs the knowledge of three elements: the frame number running in the new cell; the time slot assigned on the new channel; the timing advance. In the

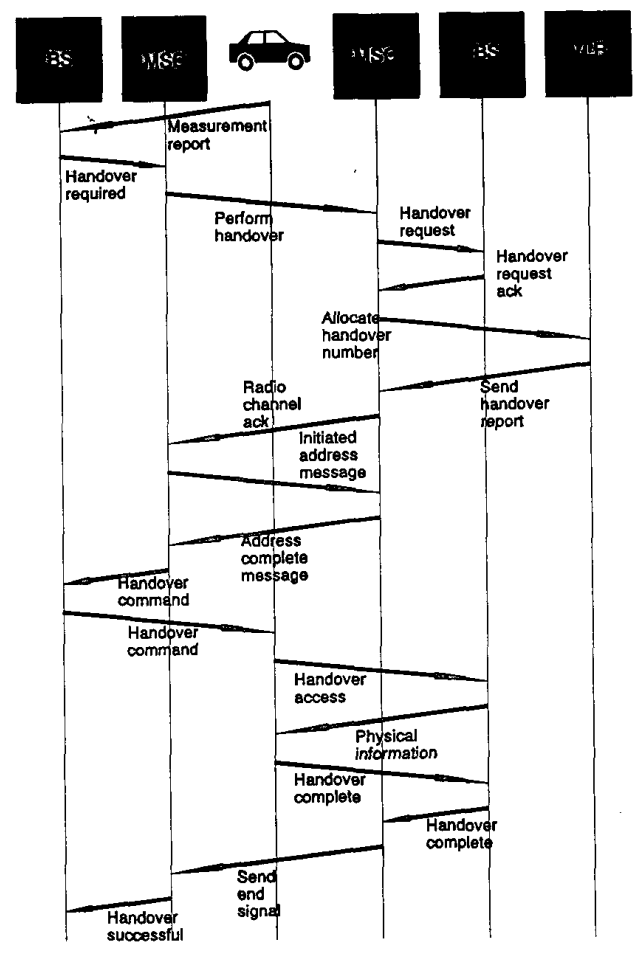

Fig. 6. Handover involving two MSC's.

GSM, the MS determines the current frame number before sending the handover access bursts, listening to the $\mathrm{BCCH}$ of the destination cell; the assigned time slot is communicated by the old BS with the message handover command; instead the initial timing advance to be adopted is provided by the destination BS, after the MS has sent the handover access bursts on the assigned FACCH with a timing advance equal to zero. The cell size and the reduced length of these bursts allow them to be received by the new BS within the assigned slot and not to overlap to the following ones.

The situation is very different when the mobile accesses a spot beam. As we have seen, on the satellite link is not possible to assure the coincidence of the uplink and downlink frame numbers at both the MS sides and the FES-BS side. Let us suppose, for example, that the coincidence is fixed on the FES-BS side. So, at the MS's, the uplink hyperframe is in advance compared to the downlink one. The precise value of this advance, in general, will not be a whole number of frames. In order that the handover access bursts are received by the FES-BS within the assigned timeslot, it is necessary for the MS to know the precise value of the staggering between uplink and downlink, before carrying out the transmission of these bursts. The only available message is the handover command, by which in the GSM all the necessary information for identifying the new channel are communicated to the MS. In this message the parameter starting time is also included. The starting time consist of three octets: the first identifies the parameter, the remaining two provide the indication of the frame number, module 42432 (corresponding to 195.8 $\mathrm{s}$ of maximum delay), from which the mobile can start 
the transmission on the new channel. On the satellite link the field occupied by this parameter might be utilized to communicate the required advance to the mobile for the frame synchronization. Indicatively the staggering should be around 110 frame periods and, in any case, not larger than 115 for spot with radius that is smaller than $800 \mathrm{~km}$. Therefore, $7 \mathrm{~b}$ are sufficient to communicate the whole number of frames. However, if only the variation with respect to the minimum staggering is transmitted, the required bits are reduced. Three bits are necessary to indicate the number of timeslots to be added to the integer number of frames. Other $8 \mathrm{~b}$ are needed for the slot fraction, that may assumes values from 0 to $156 \mathrm{~b}$. All these quantities have to be calculated by the FES-BS from which the call is handed over. Note that a certain error may be tolerated on the slot fraction that must be communicated together with the other two parameters: its value will be corrected successively with the timing advance, sent by the FES-BS with the message physical information. The remaining bits of the starting time that are not used for providing the synchronization information might again be utilized to indicate the frame from which the mobile may transmit. Of course, the maximum permitted delay is shorter than $195.8 \mathrm{~s}$, which however seems a rather large value.

It is notable that some GSM codes for parameter identification are still available: it is possible to define a new appropriate parameter to communicate the required advance on the satellite link.

Analogous considerations might be fulfilled if a staggering between uplink and downlink is assumed at the FES-BS side.

3) Handover from Spot Beam to GSM Cell: During the GSM procedure, the BS which passes the call sends the message handover required to the MSC. This message includes a list of candidate cells and information about the signal quality received from the candidate cells of the list. Each cell is identified through the BCCH carrier frequency and the BSIC. So, to identify uniquely all the cells adjacent to the serving one it is necessary for them to be characterized by a different couple BSIC-BCCH frequency. But cells with the same BSIC and BCCH frequency might be inside the spot beam. So, if such a list were used in the handover from a beam to a cell, it would not be possible to identify uniquely the candidate cells. As a solution, only the interbeam handover may be proposed when the MS communicates through the satellite. Otherwise, it is up to the mobile to allow the satellite network identify its position inside the beam: this may be achieved with the message measurement report, sent periodically by the MS. In this message the identity of at least one cell, among those reported by the mobile, must be indicated in full. In the GSM, each cell transmits its complete identification on the BCCH. Obviously, it is suitable that the specified cell is the one from which the received signal strength is the highest, since, presumably, the mobile is in that cellular site. In the measurement report less than 17 octets are used for the measurement transmission and for the identification of the serving cell and the adjacent ones. Indicating in full the identity of one cell, the message will include information about a smaller number of cells. In the GSM a cell in its location area is characterized by a two- octet code, while the location area identification requires five octets. Considering the measurement field width, the message should allow the terminal to communicate the information relating the cell where it is and three near cells, supposing that each of these latter ones is identified by the BSIC and the $\mathrm{BCCH}$ frequency. Recall that, in the GSM, the neighboring cell $\mathrm{BCCH}$ frequencies to be monitored are communicated to the mobile through the $\mathrm{BCCH}$ allocation list, transmitted on the SACCH of the serving cell. Analogously, when the mobile communicates through the satellite, in this list the FES-BS should indicate the frequencies that are $\mathrm{BCCH}$ carriers in the area where the MS is at the time. In other words, the FESBS should adapt the transmitted list in accordance with the position of the MS in the spot. This problem may be solved in the following way. Suppose that the mobile knows the GSM cell where it is immediately before the set-up. Soon after the set-up, it can communicate this information to the FES-BS, using the message measurement report only for transmitting this indication. Knowing the MS position, the FES-BS can send a $\mathrm{BCCH}$ allocation list, on the $\mathrm{SACCH}$, where only the frequencies that are $\mathrm{BCCH}$ carriers in that zone are indicated. During the communication the list will vary according to the MS position, which is determined by the FES-BS on the basis of the cell explicitly indicated in the measurement report. Note that if the cell indication is missing at the beginning of the communication, the procedure cannot start. This condition excludes a handover from satellite to GSM, when the setup occurs outside the GSM coverage. Instead the mobile can precise the cell when it accesses the satellite owing to the traffic overload in the cellular link. Modifications have to be foreseen for the message handover candidate enquiry also, sent by the satellite MSC to the FES-BS: in this message the MSC has to indicate in full the GSM cell to which it intends to pass the calls.

Till now, all the modifications to the GSM protocols affect the mobile terminal or the satellite system. But the GSM network will be interested too. Note that an MSC area overlaps to the neighboring MSC areas only with the boundary cells of its coverage. So an inter-MSC handover should occurs only between boundary cells of two MSC's. The implementation of the terrestrial network might take into account this fact. Instead, in order to hand over the calls from the satellite to the GSM, all the cells inside an MSC area should be considered boundary cells with respect to the area covered by the satellite MSC. The exploitation of this feature depends on the national implementation of the GSM, but broadly speaking does not seem easily feasible.

\section{CONCLUSIONS}

1) Summarizing Tables: The results of the study are synthetically reported in the following summarizing Tables I and II. Each procedure has been associated with one "feasibility class":

modifications to the GSM protocols are required only at the MS

modifications to the GSM standards

affect the MS and the satellite network 
TABLE I

Operational Procedures on the Satellite LinK

Selection and reselection

Synchronization

Location updating

Set-up

Double set-up from a spot beam to a spot beam: mobile originated call

Double set-up from a spot beam to a spot beam: mobile terminating call

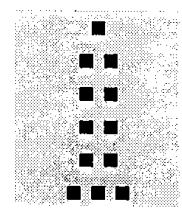

TABLE II

INTERWORKING Procedures

Double set-up from a GSM cell to a spot beam: mobile originated call

Double set-up from a GSM cell to a spot beam: mobile terminating call

Handover from a spot beam to a GSM cell

Handover from a GSM cell to a spot beam

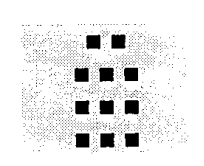

changes in the GSM network are

necessary

Note that the synchronization is performed during the location updating and the call set-up. This occurrence influences the feasibility evaluation of these two procedures. The definition "double set-up" refers to a subsequent attempt after the first one is failed.

2) Final Considerations: The reuse of the GSM standards, as far as possible, in a satellite system involves two aspects:

- the ability of the GSM to work in a different environment, compared to that one for which the standard has been designed;

- the possibility of good cooperation with the GSM cellular network.

As a response to the first issue, the procedures of the GSM seem largely re-usable in the satellite subsystem, even if some modifications are often necessary at lower OSI layers (in some aspects of the access procedures, for instance).

The interworking between satellite and cellular network could be assured by means of the "double set-up" on the satellite link when an overload occurs in the GSM and the inter-network handovers.

The double set-up for a mobile originated call involves the mobile terminal only, in the procedure outlined. This solution is in keeping with the GSM recommendations, but it is not be best one. It would be better, when cellular channels are not available, that the BS signals this situation to the mobile terminals, broadcasting a suitable parameter; then the mobile terminals should switch to the satellite band. This feature avoids a useless call set-up in the cellular network.

The handover from a GSM cell to a spot beam could permit a call to be continued when the MS is leaving the cellular coverage, still inside a spot. The implementation of this procedure leads to modify the BS's. However, as this handover mainly concerns the cells that mark the limit of the cellular coverage, the changes could be limited at these "boundary cells." The handover from a spot to a cell is an interesting traffic management feature as it permits to reduce the occupancy of the satellite resources. In this case, the
MSC's should be able to perform an inter-MSC handover with all their cells. Apart from that problem, the handover could be carried out according to the GSM procedures.

As a final consideration, it is to remark that the "interworking" between a cellular network and a satellite system based on the GSM standard presents certain limits. However, drawbacks and problems could bypassed with solutions simple and effective. These features encourage the efforts to define future mobile standards able to work in a satellite/terrestrial system with a higher degree of integration.

\section{REFERENCES}

[1] E. Del Re, "Satellite system integrated with the terrestrial cellular network for mobile communications," ESA STR 228, Aug. 1989

[2] R. Menolascino, E. Del Re, P. Iannucci, and F. Settimo, "Traffic management strategies in an integrated satellite/terrestrial cellular network for mobile services," in Proc. 2nd Int. Conf. Universal Personal Commun., Ottawa, Ont., Canada, Oct. 1993

[3] E. Del Re, F. Delli Priscoli, R. Menolascino, I. Mistretta, and F. Settimo, "Issues for the integration of satellite and cellular network for mobile communications," in Workshop Adv. Network and Technol. Concepts for Mobile, Micro and Personal Commun., JPL, Pasadena, CA, May 30-31, 1991

[4] W. R. Braun, D. Dzung, and W. C. Hagmann, "Compatibility between GSM and satellite systems," Final Rep. ESA Contract 7866/88/F/RD(SC), Dec. 1989

Enrico Del Re (M'78-SM'84) for a photograph and biography, please see the Guest Editorial of this JouRnal, p. 178.

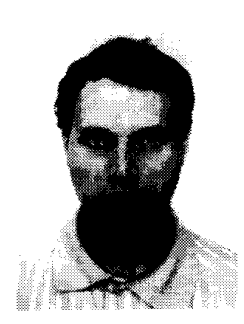

Piero Iannucci received the degree in electronic engineering from the University of Florence, Florence, Italy, in 1992. He is currently working toward the $\mathrm{Ph} . \mathrm{D}$. degree in telecommunications engineering at the University of Florence.

After his graduation, he was the recipient of a national scholarship from the Italian Space Agency (ASI) and he worked in the field of satellite personal communications. His interests include communication systems analysis and definition of protocols for personal mobile communication systems. 